\title{
An Assessment on the Performance of Reynolds Stress Model (RSM) in Modeling Churn Fully Turbulent Flow in Bubble Column Reactors
}

\author{
Bayazid Mahmoodi \\ Gas Engineering Department, \\ Petroleum University of \\ Technology,
}

Ahwaz, Iran

\author{
Mohammad R. Khosravi Nikou \\ Professor, Gas Eng. Dep., \\ Petroleum University of Technology, \\ Ahwaz, Iran
}

\author{
Masoud B. Bahrami \\ Professor, Gas Eng. Dep., \\ Petroleum University of \\ Technology, \\ Ahwaz, Iran
}

\begin{abstract}
Churn-turbulent flow of gas and liquid phase are simulated in a cylindrical bubble column reactor in laboratory scale. The model is established in a full three dimensional, unsteady state mode in an Euler-Euler multiphase modeling approach. Numerical solution of model equations is carried out by finitevolume tensor-based formulation method using open-source CFD package (OpenFOAM). The attempt is made to assess the performance and applicability of two different turbulence models named RSM and RNG k- $\varepsilon$ for modeling turbulence in churn fully turbulent flow of bubble column. For this purpose, the predictions are compared with the experimental data from literature. Performance of the turbulence models is assessed on basis of comparison of axial liquid velocity, turbulent kinetic energy and their impact on bubble breakup predictions. It is found that simulated data using RSM model has better agreement with the experimental data in comparison with RNG $\mathrm{k}-\varepsilon$.
\end{abstract}

\section{Keywords}

Bubble column, Turbulence, Hydrodynamics, CFD, OpenFOAM

\section{INTRODUCTION}

Bubble column reactors that are simple gas-liquid contactors are key component of many chemical and biochemical industries. Bubbly flows occur in a variety of industrial processes, such as Gas to liquid (GTL) in Fischer-Tropsch synthesis, manufacture of fine chemicals, oxidation reactions, coal liquefaction, and fermentation reactions. This devices widely used in industrial processes because they have simple construction withno mechanically moving parts which results in easy maintenance and low operating costs also they have good heat and mass transfer performances. In all these applications, the final product quality depends sensibly on the quality of the dispersed flow. Thus the investigation of twophase churn fully turbulent flows is of the great importance in understanding the design and the operation of many frequently used industrial gas-liquid contacting devices and it is essential to develop accurate computational flow models to understand such complex interactions and to facilitate design scale-up tasks. There are several approaches regarding the simulation of multiphase flows such as Euler-Lagrange, VOF and Eulerian-Eulerian but due to computational requirement application of preceding two methods are restricted to a few bubbles [2]. However, churn-turbulent flows lay much beyond these applicability limits, thus for such cases, it is necessary to use an Eulerian-Eulerian approach, which invokes extensive modeling to simulate the behavior of gas-liquid dispersions with high gas volume fractions. The bubbles and liquid phases are related through interaction forces that are described as drag, lift, virtual mass and turbulent dispersion forces but it is generally agreed that drag force is largely predominant over other contributions [3,4 and 5]. Due to presence of turbulence in most applications of bubble columns and its important impact on hydrodynamic of bubble column, two-phase turbulence modeling has to be considered in detail. The twoequation $\mathrm{k}-\varepsilon$ turbulence closures used in many studies $[3,6,7,8,9$ and 10] and there is several formulations(standard, realizable and RNG) of two-phase $k-\varepsilon$ equations in the literatures but based on studies by [3] only RNG type gives reasonable results in the churn fully turbulent flow regimes. Moreover, the $\mathrm{k}-\varepsilon$ model employs an isotropic description of turbulence and therefore may not be well suited to flows in which the anisotropy of turbulence significantly affects the mean flow and then all of these closures are subjected to uncertainties when used in churn turbulent flow. Although the results of gas holdup and velocity field prediction from the simulations by this models are in good agreement with experimental data but this models underestimate the turbulence fields [ 4 and 11]. The more accurate and elaborate turbulence model, RSM (Reynolds Stress Model), is one way to rectify this and some other limitations of eddy viscosity models that directly predict Reynolds stresses from their governing equations without using the eddy viscosity concept. RSM was applied by some researchers [12] for bubbly flow regimes and in the case of low gas superficial velocity of about $0.02 \mathrm{~m} / \mathrm{s}$ they approached in adequate realistic simulation results but the applicability of this model does not assessed for churn fully turbulent regime till yet.For modification of the effect of dispersed phase on single phase turbulent models, three viscosity models have been proposed and compared in the literature: the addition of a bubble induced turbulence viscosity term [13], a pseudo-bubble induced turbulence tensor [14] and the addition of bubble induced turbulence source terms in the turbulence transport equations [15,16, 17 and 8]. It is found that the addition of bubble induced turbulence source terms is the best compromise between precision and complexity [18] and therefore it is applied in the present work. In this research fully three dimensional CFD simulations of bubble-column flows in an Eulerian multi-fluid framework are conducted and addresses only the case of a single bubble size modeling aims at assessment of performance of RSM turbulence models for churn turbulent flow regime. For the validation of the proposed turbulence model the simulation results compared with the experimental data from the literature [19]. All of the 
simulations are performed using open source CFD package named OpenFOAM.

\section{SIMULATION PROCEDURE}

\subsection{Multiphase modeling}

Multiphase Eulerian-Eulerian approach which is based on ensemble-averaged mass and momentum transport will be applied in derivation of flow field equations for each phase. In the absence of interphase mass transfer terms the Reynoldsaveraged conservation equations can be written for each phase as:

Continuity equation:

$\frac{\partial}{\partial t}\left(\rho_{\varphi} \alpha_{\varphi}\right)+\nabla \cdot\left(\rho_{\varphi} \alpha_{\varphi} \mathbf{U}_{\varphi}\right)=0(1)$

Momentum equation:

$\frac{\partial}{\partial t}\left(\rho_{\varphi} \alpha_{\varphi} \mathbf{U}_{\varphi}\right)+\nabla\left(\rho_{\varphi} \alpha_{\varphi} \mathbf{U}_{\varphi} \otimes \mathbf{U}_{\varphi}\right)=\boldsymbol{\nabla} \cdot\left(\rho_{\varphi} \boldsymbol{\tau}_{\varphi}\right)+$

$\boldsymbol{\nabla} .\left(\rho_{\varphi} \mathbf{\tau}_{\varphi}^{T}\right)-\alpha_{\varphi} \boldsymbol{\nabla} p+\rho_{\varphi} \alpha_{\varphi} \mathbf{g}+\mathbf{F}_{\varphi}^{D}+\mathbf{F}_{\varphi}^{\text {none }-D}(2)$

Where $\varphi$ is the phase index and can be "g" for gas phase and

"l" for the liquid phase.The $\boldsymbol{\tau}_{\varphi}$ term that appears in the right side of equation (2) is due viscous stresses and $\boldsymbol{\tau}_{\varphi}^{t}$ is the Reynolds stress tensor that arises from the fluctuating velocities $U_{i, \varphi}^{\prime}$ of phase $\varphi$ and they can be written as:

$\boldsymbol{\tau}_{\varphi}=-\alpha_{\varphi} \mu_{\varphi}\left[\left(\boldsymbol{\nabla} \mathbf{U}_{\varphi}+\left(\boldsymbol{\nabla} \mathbf{U}_{\varphi}\right)^{T}\right)-\frac{2}{3}\left(\boldsymbol{\nabla} \cdot \mathbf{U}_{\varphi}\right) \mathbf{I}\right]$,

$\boldsymbol{\tau}_{\varphi}^{t}=-\alpha_{\varphi} \mathbf{R}_{\varphi}, \mathbf{R}_{\varphi}=R_{i j, \varphi}=\overline{U_{i, \varphi}^{\prime} U_{j, \varphi}^{\prime}}$

The $\boldsymbol{F}_{k}^{D}$ is standard drag force that acts on bubble surface and $\boldsymbol{F}_{k}^{\text {none }}-D$ represents all the none drag interphase coupling terms except pressure. Drag force is the predominant interfacial interaction force in gas-liquid flows of bubble columns $[4,5]$. Based on the studies by [3] the magnitude of the drag force is more than 100 times that of the other forces. Consequently the drag force is only interphase force that is accounted for in transport equations in this work with a significantly higher magnitude than added mass, lift and turbulent dispersion. Commonly the drag force represented as $\mathbf{F}_{k}^{D}=\frac{3}{4} C_{D} \frac{\alpha_{\mathrm{g}} \rho_{l}}{d_{\mathrm{g}}}\left|\mathbf{U}_{r}\right| \mathbf{U}_{r}, \quad \mathbf{U}_{r}=\mathbf{U}_{l}-\mathbf{U}_{g} \quad$ (5)where $C_{D}$ is drag force coefficient that is retrieved from the following [20] relation:

$C_{D}=\left\{\begin{array}{r}\frac{24\left(1+0.15 R e_{d}^{0.687}\right)}{R e_{d}} R e_{d}<1000 \\ 0.44 \quad R e_{d}>1000\end{array}\right.$

Where the $R e_{d}$ in the above relation is dimensionless Reynolds number and represented as:

$$
R e_{d}=\frac{U_{\mathrm{g}} d_{b}}{v_{l}}
$$

Where $d_{b}$ is the single bubble diameter that is considered by the following equation [21]:

$d_{b}=3 \mathrm{~g}^{0.44} \gamma^{0.34} \mu_{l}^{0.22} \rho_{l}^{-0.45} \rho_{\mathrm{g}}{ }^{-0.11} U_{\mathrm{g}}{ }^{-0.02}(7)$

\subsection{Turbulence closure}

Presence of Reynolds stress tensor in time-averaged transport equations demands application of additional closure relations for turbulence modeling. Reynolds stress tensor can be modeled based on Boussinesq hypothesis:

$\mathbf{\tau}_{\varphi}^{t}=-\alpha_{\varphi} v_{\varphi}^{t}\left[\left(\boldsymbol{\nabla} \mathbf{U}_{\varphi}+\left(\boldsymbol{\nabla} \mathbf{U}_{\varphi}\right)^{T}\right)-\frac{2}{3}\left(\boldsymbol{\nabla} \cdot \mathbf{U}_{\varphi}\right) \mathbf{I}\right]-\alpha_{\varphi} \frac{2}{3} k \mathbf{I}(8)$

In this case $v_{\varphi}^{t}$ can be retrieved by well-known single phase $k-\varepsilon$ models derived by [22] as it is the case in many studies $\left[23,16,24,4\right.$ and3]. The $v_{\varphi}^{t}$ will be calculated by following relation:

$v_{\varphi}^{t}=C_{\mu} \frac{k_{\varphi}^{2}}{\varepsilon_{\varphi}}$

Where $C_{\mu}$ is a constant and its value is 0.09 .
And $k_{\varphi}$ and $\varepsilon_{\varphi}$ is calculated by the RNG $k-\varepsilon$ transport equations as:

$\frac{\partial}{\partial t}\left(\alpha_{\varphi} k_{\varphi}\right)+\nabla \cdot\left(\alpha_{\varphi} \mathbf{U}_{\varphi} k_{\varphi}\right)=\nabla \cdot\left(\alpha_{\varphi}\left(\frac{v_{\varphi}+v_{\varphi}^{T}}{\sigma_{k}}\right) \nabla k_{\varphi}\right)+$

$\alpha_{\varphi}\left(G_{\varphi}-\varepsilon_{\varphi}\right)+S_{k_{\varphi}}^{B I}(10)$

$\frac{\partial}{\partial t}\left(\alpha_{\varphi} \varepsilon_{\varphi}\right)+\boldsymbol{\nabla} \cdot\left(\alpha_{\varphi} \mathbf{U}_{\varphi} \varepsilon_{\varphi}\right)=\boldsymbol{\nabla} \cdot\left(\alpha_{\varphi}\left(\frac{v_{\varphi}+v_{\varphi}^{T}}{\sigma_{\varepsilon}}\right) \nabla \varepsilon_{\varphi}\right)+$

$\alpha_{\varphi} \frac{\varepsilon_{\varphi}}{k_{\varphi}}\left(C_{\varepsilon, 1} G_{\varphi}-C_{\varepsilon, 2} \varepsilon_{\varphi}\right)-\alpha_{\varphi} R_{\varepsilon, \varphi}+S_{\varepsilon_{\varphi}}^{B I}(11)$

In which source terms are shown in the following:

$G_{\varphi}=2 v_{\varphi}^{T} \mathbf{S}_{\varphi}^{2}, \mathbf{S}_{\varphi}=\operatorname{symm}\left(\nabla \mathbf{U}_{\varphi}\right)$

$R_{\varepsilon, \varphi}=\frac{C_{\mu, \varphi} \eta_{\varphi}^{3}\left(1-\frac{\eta \varphi}{\eta 0}\right)}{1+\beta_{0} \eta_{\varphi}^{3}} \frac{\varepsilon_{\varphi}^{2}}{k_{\varphi}}, \eta_{\varphi}=\sqrt{2 \mathbf{S}_{\varphi}^{2}} \frac{k_{\varphi}}{\varepsilon_{\varphi}}(13)$

The additional terms $S_{k_{\varphi}}^{B I}$ and $S_{\varepsilon_{\varphi}}^{B I}$ in the above equations accounts for the effect of bubbles on liquid phase and was modeled by the following relations [8 and 25]

$S_{k_{\varphi}}^{B I}=\alpha_{l} C_{k}\left|\mathbf{F}_{\varphi}^{D}\right|\left|\mathbf{U}_{r}\right|$

$S_{\varepsilon_{\varphi}}^{B I}=\frac{C_{\varepsilon, 1} C_{\varepsilon}}{C_{k}} \cdot \frac{\varepsilon_{l}}{k_{l}} S_{k_{\varphi}}^{B I}$

All of the constants that are used in RNG turbulence model relations are shown in Tab 1.

Table 1.RNG turbulence model constants

\begin{tabular}{|l|c|c|c|c|c|c|}
\hline constant & $C_{k}$ & $C_{\varepsilon, 1}$ & $C_{\varepsilon, 2}$ & $C_{\varepsilon}$ & $\sigma_{k}$ & $\sigma_{\varepsilon}$ \\
\hline value & 1.44 & 1.44 & 1.92 & 1 & 1 & 1.3 \\
\hline
\end{tabular}

The Reynolds stress tensor also can be directly retrieved from more sophisticated RSM models as it is the case in some other studies [12, 26 and 27]. The transport equations for Reynolds stress tensor is as:

$$
\begin{aligned}
& \frac{\partial\left(\alpha_{\varphi} \mathbf{R}_{\varphi}\right)}{\partial t}+\boldsymbol{\nabla} \cdot\left(\alpha_{\varphi} \mathbf{U}_{\varphi} \mathbf{R}_{\varphi}\right)=\alpha_{\varphi} \mathbf{P}_{\varphi}+\alpha_{\varphi} \emptyset_{\varphi}- \\
& \boldsymbol{\nabla} \cdot\left(\alpha_{\varphi}\left(v_{\varphi} \mathbf{I}+\frac{2}{3} C_{s} \frac{k_{\varphi}}{\varepsilon_{\varphi}} \mathbf{R}_{\varphi}\right) \boldsymbol{\nabla} \mathbf{R}_{\varphi}\right)-\frac{2}{3} \varepsilon_{\varphi} \mathbf{I}+\mathbf{S}_{R_{\varphi}}^{B I}(16)
\end{aligned}
$$

The source terms in the above equation are given as:

$$
\begin{aligned}
& \mathbf{P}_{\varphi}=-2 \operatorname{symm}\left(\mathbf{R}_{\varphi} \cdot \nabla \mathbf{U}_{\varphi}\right) \\
& \emptyset_{\varphi}=-C_{1} \frac{\varepsilon_{\varphi}}{k_{\varphi}}\left(\mathbf{R}_{\varphi}-\frac{2}{3} k_{\varphi} \mathbf{I}\right)-C_{2} \operatorname{dev}\left(\mathbf{P}_{\varphi}\right) \\
& k_{\varphi}=\frac{1}{2} \operatorname{tr}\left(\mathbf{R}_{\varphi}\right) \\
& \mathbf{S}_{R_{\varphi}}^{B I}=\frac{2}{3} S_{k_{\varphi}}^{B I} \mathbf{I}(19)
\end{aligned}
$$

The $\varepsilon_{\varphi}$ in the above relations will be retrieved from the following transport equation:

$$
\begin{aligned}
& \frac{\partial\left(\alpha_{\varphi} \varepsilon_{\varphi}\right)}{\partial t}+\boldsymbol{\nabla} \cdot\left(\alpha_{\varphi} \mathbf{U}_{\varphi} \varepsilon_{\varphi}\right)=\alpha_{\varphi} \frac{\varepsilon_{\varphi}}{k_{\varphi}}\left(C_{\varepsilon 1} G_{\varphi}-C_{\varepsilon 2} \varepsilon_{\varphi}\right)+ \\
& \boldsymbol{\nabla} \cdot\left(\alpha_{\varphi}\left(v_{\varphi} \mathbf{I}+C_{\varepsilon} \frac{k_{\varphi}}{\varepsilon_{\varphi}} \mathbf{R}_{\varphi}\right) \boldsymbol{\nabla} \varepsilon_{\varphi}\right)+S_{\varepsilon_{\varphi}}^{B I}(20)
\end{aligned}
$$


All of the constants that are used inRSM turbulence model relations are shown in Tab 2.

Table 2.RSM turbulence model constants.

\begin{tabular}{|l|c|c|c|c|c|c|}
\hline constant & $C_{1}$ & $C_{2}$ & $C_{s}$ & $C_{\varepsilon 1}$ & $C_{\varepsilon 2}$ & $C_{\varepsilon}$ \\
\hline value & 1.8 & 0.6 & 0.22 & 1.45 & 1.83 & 0.18 \\
\hline
\end{tabular}

Liquid phase turbulence is modeled through RNG and Reynolds stress model transport equations as cited above, while the turbulence in dispersed gas phase is predicted through correlations from the theory of dispersion of discrete particles by the homogeneous turbulence [28].

\subsection{Numerical simulation}

In order to perform numerical simulation, Open source Field Operation and Manipulation (OpenFOAM) C++ libraries are used. OpenFOAM is a one of the first major scientific packages that is written in $\mathrm{C}++$ due to its highest modularity and object oriented features and its code is released as free and open source software under the GNU General Public License. OpenFOAM is supplied with numerous preconfigured solvers, utilities and libraries and gives its users the capability to development of customized numerical solvers, and pre-/post-processing utilities for the solution of continuum mechanics problems, including computational fluid dynamics (CFD).

The set of conservation equations have been discretized in time and spatial coordinate using finite-volume method following the same procedure that has been adapted by [29]. A wide range of discretization schemes are available in openFoam to choose. Eulerian first order discretization scheme was applied for the time derivatives. Linear Gaussian integration that is based on summing value on cell faces which is interpolated from cell centers, is chosen for gradient operators. High order bounded discretization scheme, limited linear differencing scheme which is developed by [30], is applied for discretization of convective terms. Each set of resulting algebraic equations is solved in segregated manner as follows:

1. Continuity equation for gas phase will be solved and the phase fraction of the liquid phase will be retrieved from equation $\alpha_{\mathrm{g}}+\alpha_{l}=1$.

2. Drag forces will be calculated and momentum equation will be solved for each phase.

3. Pressure-velocity coupling will be implemented using PISO solution algorithm which requires a momentum predictor and a correction loop in which the pressure equation is solved and the momentum corrected based on the pressure change.

4. Material derivatives of velocity field for each phase will be updated.

5. Turbulence field equations will be solved.

Different iterative methods are used for the solution of sparse matrixes which resulted from the set of discretized model equations on the discretized solution domain based on the structure of those matrixes. Generalized geometric-algebraic multi-grid (GAMG) solver with diagonal incompleteCholesky (DIC) smoother is used for the symmetric pressure equation and preconditioned bi-conjugate gradient (PBiCG) with diagonal incomplete-LU (DILU) preconditioner solver is used for other asymmetric equations. In this work the original two phase Eulerian solver of OpenFOAM (twoPhaseEulerFoam) had been customized with the capability of using other turbulence models. For this purpose the new applications rngTwoPhaseEulerFoam (solver that uses RNG $k-\varepsilon$ model) and rsmTwoPhaseEulerFoam (solver that uses RSM model) are developed.

\section{RESULTS AND DISCUSSION}

The numerical simulation of two phase (water-air) flow with an Eulerian-Eulerian modeling approach had been carried out for a laboratory scale cylindrical vessel in the same physical conditions that was used in experimental works [19]. The experimental vessel specifications are shown in Tab 3.Due to the small scale of the holes of the sparger and limitation of computational resources the inlet holes was not resolved and the gas phase introduced uniformly with superficial velocity $0.096 \mathrm{~m} / \mathrm{s}$ in the bottom of the column and $\alpha_{g}$ is set to 1 to only gas enters the column. All the walls are treated as nonslip boundaries for momentum equations and standard wall function are applied for turbulence fields and an atmospheric condition is imposed in the column top. In order to the simulation results quantitatively be comparable with experimental data initially we set the same conditions for our simulations as reported in experiments. Thus we set the $\alpha_{l}=1$ up to the static level of the water in experiment and above that a gas-zone ( $\alpha_{l}=1$ ) was set up to a level about $100 \%$ static level of liquid in column to prevent the liquid from escaping the vessel.

Table 3. Column size, sparger design, operating conditions

\begin{tabular}{|l|l|}
\hline Column diameter $(\mathrm{m})$ & 0.14 \\
\hline Superficial gas velocity $(\mathrm{m} / \mathrm{s})$ & 0.096 \\
\hline Static liquid height $(\mathrm{m})$ & 0.98 \\
\hline Dynamic liquid height $(\mathrm{m})$ & 1.23 \\
\hline Sparger porosity $(\%)$ & 0.05 \\
\hline Size of spargerholes $\left(\times 10^{-4}\right)$ & 4 \\
\hline
\end{tabular}

Computational grid is based on the unstructured set of blocks each containing structured grid. In order to set the simulation results grid independent a systematic mesh study carried out as studied by [3]. Different mesh sizes ranges from 43520 to 107520 cells had been investigated and finally in the present work a non-uniform structured grid of 85,732 (with element size: $z=1 \mathrm{~cm}, r=0.7 \mathrm{~cm}, \theta=1 \mathrm{~cm}$ and wall refinement) cells was used for all the simulated cases. A sample of the meshes used in this work is shown in Figure 1.

Figure 2 shows the instantaneous iso-surfaces of the gas volume fraction in columns for simulation results using RNG and RSM turbulence models. The plots show the threedimensional spiral structures of bubble plumes continuously ascending in the center of column carrying the surrounding liquid upward and results in recirculating of liquid downwards alongside the wall. In fact the real observed bubble column flows are highly transient and turbulent with rising threedimensional vortical bubble swarms and the flow never reaches steady-state but time averaging produces reproducible stationary patterns. Thus in order to compare the simulated data with experimental results, radial profiles of timeaveraged fields have been tangentially and axially averaged over the fully developed region of the flow (between $\mathrm{z}=0.18 \mathrm{~m}$ and $\mathrm{z}=1.04 \mathrm{~m})$. 


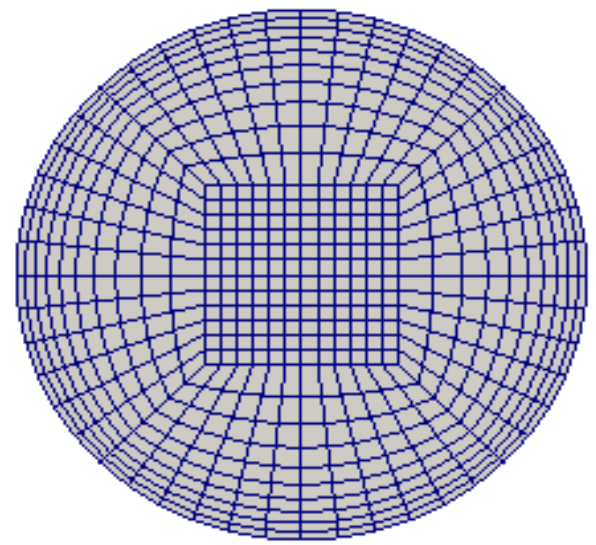

Fig1: Bubble column geometry and mesh used in this work.

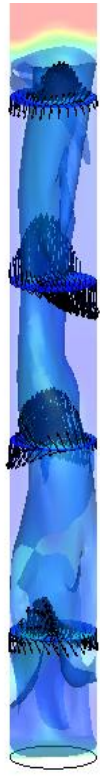

A

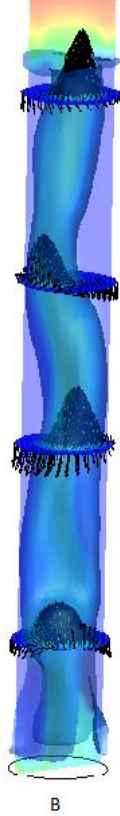

B

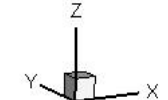

0.929293 0.848485 0.757576
0.676768 0.69596 0.505051 0.424242 0.343434 0.252525 0.0909091

Fig2: Snapshots of the instantaneous iso-surfaces of the gas holdup and liquid velocity vectors in different lateral cross sections of the column: (A) RNG $k-\varepsilon$, (B) RSM.

Figure 3 show the profiles of the time- averaged nondimensional mean axial liquid velocity, that is, $\left\langle U_{z}\right\rangle / U_{\mathrm{g}}$ asa function of $\mathrm{r} / \mathrm{R}$ for both RNG $\mathrm{k}-\varepsilon$ and RSM models. As can be seen from the figure, for both models, the general shape of velocity well captured and the discrepancy between the simulated and experimental profiles of liquid axial velocity are negligible except in the core region of the column where in churn turbulent flows the bubble interactions (breakup and coalescence) results in a wide range of bubble size distributions while in this work a single size diameter had been used. Figure 4 shows comparison of model prediction for the turbulent kinetic energy rate with the experimental data. As can be seen, the experimental trend (i.e., an increase from the core to the middle and preceding decrease to the wall) of the turbulent kinetic energy rate are predicted by both models. But RNG model under predicts the real magnitude of turbulence kinetic energy and it is due to isotropic turbulence modeling assumption of two-equation models that is not valid for modeling of anisotropic turbulence in churn fully turbulent flow.

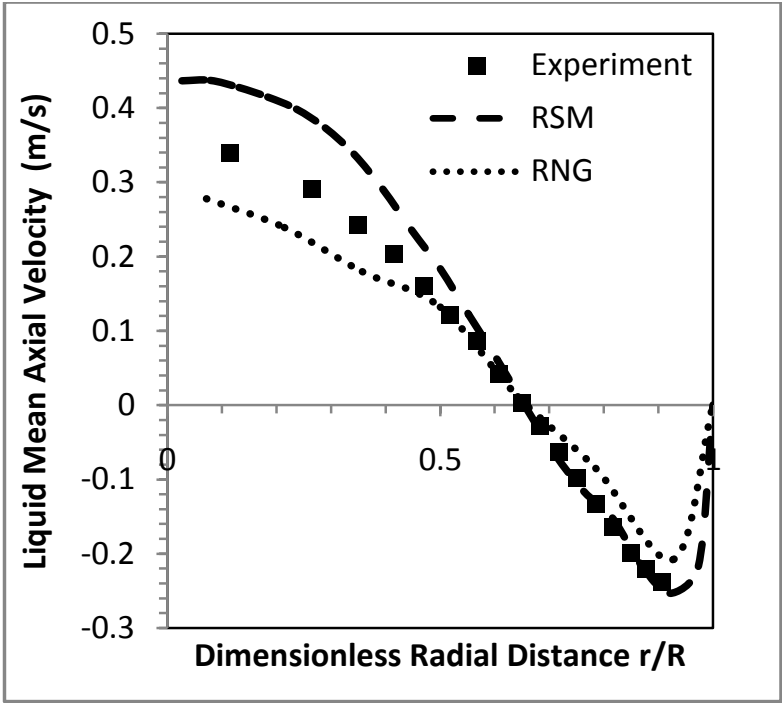

Fig3: Comparison of tangential and axial averaged radial profiles of Simulated and Experimental time-averaged liquid axial velocity over the fully developed region of flow.

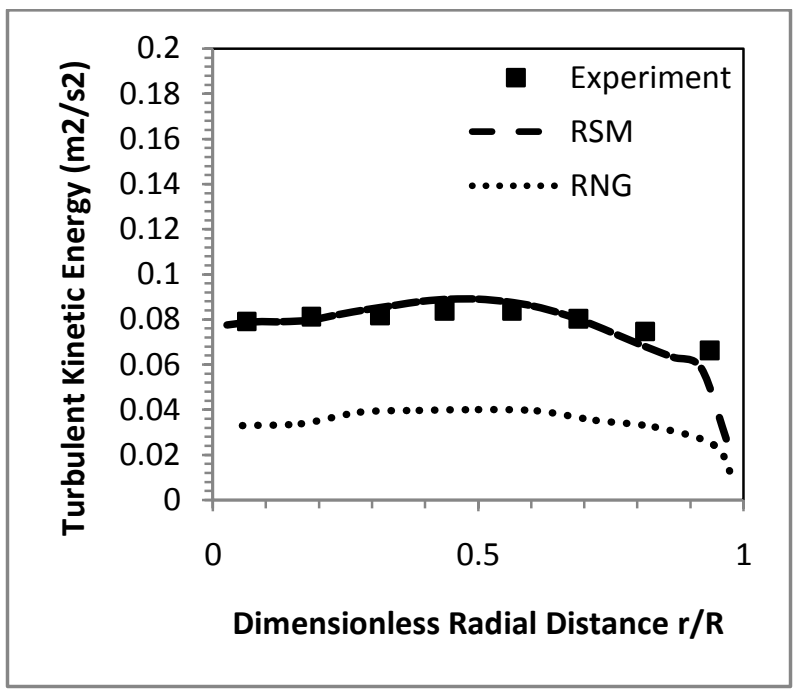

Fig4: Comparison of tangential and axial averaged radial profiles of Simulated and Experimental time-averaged liquid turbulent kinetic energy over the fully developed region of flow.

One of the main issues in modeling bubble population balanceis in the inability of turbulence models to predict accurate the level of turbulent fields that resulted from under prediction of breakage using breakup models [4 and 11]. The relevance of the various turbulence models can be further discussed through the comparison of predictions of breakage using two of the commonly used breakage kernels [31]: $\Omega(v)=$

$0.923 K_{1}\left(1-\alpha_{g}\right)\left(\frac{\varepsilon_{l}}{d^{2}}\right)^{\frac{1}{3}} \int_{0}^{0.5} \int_{\xi_{\min }}^{1} \frac{(1+\xi)^{2}}{\xi^{\frac{11}{3}}} \exp (-\varphi) d f_{v} d \xi$

Where the dimensionless parameters in above equations are as following:

$\psi=\frac{12 C_{f} \gamma_{l}}{\beta \rho_{l} \varepsilon_{l}^{\frac{2}{3}} d^{\frac{5}{3}} \xi^{\frac{11}{3}}}$,

$C_{f}=f_{v^{3}}^{\frac{2}{3}}+\left(1-f_{v}\right)^{\frac{2}{3}}-1$, 
$\xi_{\text {min }}=11.4\left(\frac{v_{l}^{3}}{\varepsilon_{l}}\right)^{\frac{1}{4}} / d_{\text {min }}$

And [32]:

$\Omega(v)=\frac{K \sqrt{\beta\left(\varepsilon_{l} d\right)^{\frac{2}{3}}-12 \frac{\gamma_{l}}{\rho_{l} d}}}{d}, \quad K=0.25, \beta=8.2$



Fig5a: Comparison of tangential and axial averaged radial profiles of Luo and Svendsen breakup model in different bubble diameters for case RNG(lower surface) and RSM(upper surface) over fully developed region of flow.

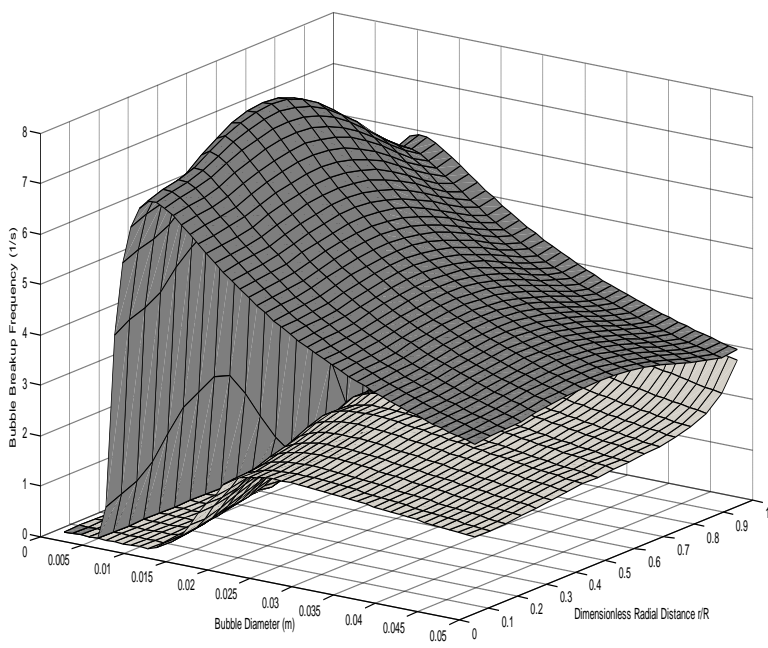

Fig 5b: Comparison of tangential and axial averaged radial profiles of Martinez-Bazan breakup model in different bubble diameters for case RNG(lower surface) d RSM(upper surface) over fully developed region of flow.

Figure 5 shows tangentially and axially averaged breakup model predictions in different bubble diameters over the fully developed region of flow. As can be seen from the Figure, both of the breakup models in the case of RSM turbulence model predict higher levels of breakup rate. The importance of this effect can be discussed by the fact that former researchers [1] that used $\mathrm{k}-\varepsilon$ turbulence model incorporating the population balance model for bubble size modeling found that quantitative agreements between the experimental data and simulations are obtained for the time-averaged axial liquid velocity and the turbulent kinetic energy profiles, only when model predicted bubble breakup rate is increased about ten times to balance the bubble coalescence rate. Thus the application ofRSM turbulence model can address the issue of bubble breakup modeling in bubble columns in much better levels than RNG $\mathrm{k}-\varepsilon$ model and it is a step forward towards a better description and modeling of churn fully turbulent flows in bubble columns.

\section{CONCLUSION}

Fully Three-dimensional unsteady simulations of churn fully turbulent gas-liquid flow in the bubble columns has been carried out using the Euler-Euler approach. The performance of Reynolds stress model (RSM) in comparison with RNG k$\varepsilon$ turbulence model have been examined through comparison with experimental data [19]. The predicted liquid velocity profile and turbulent kinetic energy profile for RSM turbulence model are in good agreement with the available data. Although RNG $\mathrm{k}-\varepsilon$ model also perform reasonable, if objective is to understand the steady and time-averaged features of the flow but RSM models better accuracy in the representation of turbulence phenomena could also positively affect other aspects of the physical model such as better estimation of break-up rates during the implementation of bubble-population balance.

\section{SYMBOLS USED}

$C_{\mu} \mathrm{k}-\varepsilon$ constant (0.09), dimensionless

$\mathrm{d} \quad$ bubble diameter, $\mathrm{m}$

Finterface forces, $\mathrm{Nm}^{-3}$

g acceleration due to gravity, $m s^{-2}$

I identity matrix

$\mathrm{k} \quad$ turbulent kinetic energy, $m^{2} s^{-2}$

$\mathrm{p}$ pressure, $\mathrm{Pa}$

R Reynolds stress tensor, $m^{2} s^{-2}$

$R_{i j} \quad$ Reynolds stress tensor components, $m^{2} s^{-2}$

Re Reynolds number, dimensionless

$\mathrm{t}$ time, $\mathrm{s}$

$\mathbf{U}$ velocity vector, $m s^{-2}$

$U$ velocity magnitude, $m s^{-2}$

Greek symbols

$\alpha$ volume fraction of each phase, dimensionless

Eturbulent dissipation rate, $m^{2} s^{-3}$

$\mu$ dynamic viscosity, $\mathrm{kgm}^{-1} \mathrm{~s}^{-1}$

$v$ kinematic viscosity, $\mathrm{kgm}^{-1} \mathrm{~s}^{-1}$

$\rho$ density, $\mathrm{kgm}^{-3}$ 
$\gamma$ surface tension, $\mathrm{Nm}^{-1}$

$\sigma$ turbulent Schmidt number

$\Omega$ breakup rate $s^{-1}$

Subscripts and superscripts

1 liquid

g gas

$\varphi$ phase index

$\mathrm{t}$ turbulent

D drag

BI bubble induced

\section{REFERENCES}

[1] Chen, P., Sanyal, J., Dudukovic, M.P. 2005. Numerical simulation of bubble columns flows: effect of different breakup and coalescence closures. Chem. Eng. Sci. 60,1085-1101.

[2] Ranade, V. V. 2002. Computational flow modeling for chemical reactor engineering, Academic Press, 525 B Street, Suite 1900, San Diego, California 92101-4495, USA, , pp. 327-366.

[3] Laborde-Boutet, C., Larachia F., Dromard, N., Delsart, O., Schweich, D. 2009. CFD simulation of bubble column flows: Investigations on turbulence models in RANS approach. Chem. Eng. Sci. 64,4399-4413.

[4] Chen, P. 2004. Modeling the fluid dynamics of bubble column flows. Ph.D. Thesis, Sever Institute of Washington University, St Louis, USA.

[5] Olmos, E. 2002. Etude expérimentaleetnumérique des écoulementsgaz-liquide en colonnes à bulles. Ph.D. Thesis, Institut National Polytechnique de Lorraine, Nancy, France.

[6] Ekambara, K., Dhotre, M.T., Joshi, J.B. 2005. CFD simulations of bubble column reactors: $1 \mathrm{D}, 2 \mathrm{D}$ and 3D approach. ChemEngSci 60,6733-6746.

[7] Padial, N. T., Vander Heyden, W. B., Rauenzahn, R. M., Yarbro, S. L. 2000. Three-dimensional simulation of a three-phase draft-tube bubble column. Chem. Eng. Sci. 55,3261-3273.

[8] Pfleger, D., Becker, S. 2001. Modeling and simulation of the dynamic flow behaviour in a bubble column. Chem. Eng. Sci. 56,1737-1747.

[9] Sokolichin, A., Eigenberger, G. 1999. Applicability of the Standard $\mathrm{k}-\varepsilon$ turbulence model to the dynamic simulation of bubble columns: part I detailed numerical simulations. Chem. Eng. Sci. 54,2273-2284.

[10] Van Baten, J. M., Krishna, R. 2001. Eulerian simulations for determination of the axial dispersion of liquid and gas phases in bubble columns operating in the churnturbulent regime. Chem. Eng. Sci. 56,503-512.
[11] Jakobsen, H. A., Lindborg, H., Dorao, C. A. 2005. Modeling of bubble column reactors: progress and limitations. Industrial and Engineering Chemistry Research 44,5107-5151.

[12] Cartland Glover, G. M., Generalis, S. C. 2004. The modeling of buoyancy driven flow in bubble columns. Chem. Eng. Process. 43,101-115.

[13] Sato, Y., Sekoguchi, K. 1975. Liquid velocity distribution in two-phase bubble flow. International Journal of Multiphase Flow 1,2,79-95.

[14] Arnold, F. C. 1988. Physical model for two-phase flow in steam injection wells. American Institute of Chemical Engineers, National Meeting, New York, United States. pp.42-76.

[15] Gu, H. Y., Guo, L. J. 2005. Modelling and simulation of the dynamic flow behaviour in a rectangular bubble column. Journal of Engineering Thermophysics. 26,7275 .

[16] Mudde, R. F., Simonin, O. 1999. Two- and threedimensional simulations of a bubble plume using a twofluid model. Chem. Eng. Sci. 54,5061-5069.

[17] Olmos, E., Gentric, C., Midoux, N. 2003. Numerical description of flow regime transitions in bubble column reactors by multiple gas phase model. Chem. Eng. Sci. $58,2113-2121$

[18] Sokolichin, A., Eigenberger, G., Lapin, A. 2004 Simulation of buoyancy driven bubbly flow: established simplifications and open questions. A.I.Ch.E. Journal. 50-24.

[19] Degaleesan, S. 1997. Fluid dynamic measurements and modeling of liquid mixing in bubble columns. D.Sc. Thesis, Sever Insitute of Washington University, St Louis, USA.

[20] Schiller, L., Naumann, Z. 1935. Uber die grundlegendenBerechnungenbei der Schwerkraftaufbereitung. Zeitschrift des VereinsDeutscherIngenieure. 77,318-320.

[21] Wilkinson, P. M. 1991. Physical aspects and scale-up of high pressure bubble columns. D.Sc. Thesis, University of Groningen, The Netherlands.

[22] Launder, B. E., Spalding, D. B. 1972. The numerical computation of turbulent flows, Computer Methods in Applied Mechanics and Engineering. 3,269-289.

[23] Elghobashi, S. E., Abou-Arab, T.W. 1982. A twoequation turbulence model for two-phase flows. The Physics of Fluids. 4,26,931-938.

[24] Rusche, H. 2002. Computational fluid dynamics of dispersed two-phase flows at high phase fraction. Ph.D Thesis, Department of mechanical Engineering, Imperial College of Science, Technology and Medicine, University of London, London, UK. 
[25] Moullec, Y. L., Potier, O., Gentric, C., Leclerc, J. P. 2008. Flow field and residence time distribution simulation of a cross-flow gas-liquid wastewater treatment reactor using CFD. Chem. Eng. Sci. 63,24362449.

[26] Cokljat D., Slack M., and Vasquez S. A. 2003. ReynoldsStress Model for Eulerian Multiphase. In Y. Nagano K. Hanjalic and M. J. Tummers, editors Proceedings of the 4th International Symposium on Turbulence Heat and Mass Transfer. Begell House, Inc. 1047-1054.

[27] Ekambara, K., Dhotre M. T. 2010. CFD simulation of bubble column. Nuclear Engineering and Design 240,963-969.

[28] Tchen, C. M. 1947. Mean value and correlation problems connected with the motion of small particles suspended in a turbulent fluid. D.Sc. Thesis, TU, Delft, The Netherlands.
[29] Weller, H. G., Tabor, G., Jasak, H., Fureby, C. 1998. A tensorial approach to computational continuum mechanics using object-oriented techniques. Computers in Physics 12,6,620-631.

[30] Jasak, H., Weller, H. G., Gosman, A. D. 1999. High resolution NVD differencing scheme for arbitrarily unstructured meshes. International Journal for Numerical Methods in Fluids 31,431-449.

[31] Luo, H., Svendsen, H. F. 1996. Theoretical model for drop and bubble breakup in turbulent dispersions. A.I.Ch.E. Journal. 42,1225-1233.

[32] Martínez-Bazán, C., Montañéz, J. L., Lasheras, J. C. 1999a. On the breakup of an air bubble injected into a fully developed turbulent flow. Part 1: breakup 